\title{
Modifying the Interlayer Interaction in Layered Materials with an Intense IR Laser
}

\author{
Yoshiyuki Miyamoto, ${ }^{1}$ Hong Zhang, ${ }^{2, *}$ Takehide Miyazaki, ${ }^{1}$ and Angel Rubio ${ }^{3,4}$ \\ ${ }^{1}$ Nanosystem Research Institute, National Institute of Advanced Industrial Science and Technology (AIST), \\ Central 2, 1-1-1 Umezono, Tsukuba 305-8568, Japan \\ ${ }^{2}$ College of Physical Science and Technology, Sichuan University, Chengdu 610065, China \\ ${ }^{3}$ Max Planck Institute for the Structure and Dynamics of Matter, Luruper Chaussee 149, 22761 Hamburg, Germany \\ ${ }^{4}$ Nano-Bio Spectroscopy group and European Theoretical Spectroscopy Facility (ETSF), Universidad del País Vasco CFM CSIC-UPVI \\ EHU-MPC DIPC, 20018 San Sebastian, Spain \\ (Received 12 January 2015; published 19 March 2015)
}

\begin{abstract}
We propose a transient interlayer compression in two-dimensional compound materials by using an intense IR laser resonant with the out-of-plane optical phonon mode ( $A_{2 \mathrm{u}}$ mode). As a test case, we studied bilayer hexagonal boron nitride $(h-\mathrm{BN})$, which is one of the compound layered materials. Excited state molecular dynamics calculations using time-dependent density functional theory show an $11.3 \%$ transient interlayer contraction of $h$-BN due to an interlayer dipole-dipole attraction of the laser-pumped $A_{2 \mathrm{u}}$ mode. These results are applicable to other layered compound materials. Such layered materials are a good material for nanospace chemistry, e.g., intercalating molecules and acting with them, and IR irradiation to contract the interlayer distance could provide a new route for chemical reactions under pressure. The duration of the contraction is at least $1 \mathrm{ps}$ in the current simulation, which is observable by high-speed electron-beam diffraction measurements.
\end{abstract}

The exotic properties of layered materials, such as graphene [1], hexagonal boron nitride ( $h$-BN) [2], chalcogenide disulfide [3-5], and others [6], and their application in next-generation electronic devices $[7,8]$ have become a major focus of scientific research. $h$-BN has recently attracted much attention as insulating junctions for graphene tunneling transistors [9] and as an insulating substrate for graphene field effect transistors [10-13], which show higher performance than those on silicon oxide substrates. The electronic properties of these layered materials are very sensitive to the interlayer distance, which is governed by weak interactions such as the van der Waals force (VDW) [14]. Therefore, controlling the interlayer distance of layered materials will allow the material properties to be controlled in technological applications. Previously, ultrashort strong laser irradiation has been shown, experimentally, to induce compression and expansion of graphene layers $[15,16]$ and, theoretically, to induce the layer-by-layer exfoliation of graphite [17]. These phenomena are triggered by electronic excitation. In this Letter, on the other hand, we propose that phonon excitations cause interlayer contraction.

Controlling dynamics of charged particles with a dynamical or static electric field was a hot topic of nanoparticle science [18] as well as plasma physics, while controlling neutral matters is quite challenging as we propose here using an intense laser that usually causes structural destruction through fast local heating [19]. The laser-induced electronic excitation may also cause nonthermal melting [20]. In contrast, laser-induced strengthening of interatomic attraction was theoretically proposed for a helium dimer [21]. The mechanism of strengthening was assigned to dipole-dipole attraction due to the concerted motion of the electron cloud and nuclei driven by the optical electric field ( $E$ field).

Instead of being a process by electronic excitations, as in Ref. [21], the attraction mediated by phonon excitations has been proposed from the first-principles electron-ion dynamics calculations of the $h$-BN bilayer under IR irradiation. When anions and cations move in opposite out-of-plane directions, an electric dipole normal to each layer is induced. When the ionic movement is in the same direction in all layers, parallel dipoles are induced in each layer causing interlayer attraction. Such out-of-plane lattice vibration is possible with light with an optical frequency resonant with the vibration. We expect that our results will also be applicable to other two-dimensional ionic materials such as transition metal dichalcogenides.

Figure 1(a) shows bilayer $h$-BN. Each layer of $h$-BN has a honeycomb lattice structure with two sublattices in the hexagonal unit cell, which are occupied by boron (B) (cation) and nitrogen $(\mathrm{N})$ (anion) atoms. $\mathrm{N}(\mathrm{B})$ atoms in a layer are located directly above the $\mathrm{B}(\mathrm{N})$ atoms of the neighboring layer. $h$-BN is a wide-gap insulator and is transparent to IR laser light. However, IR laser light with a polarization vector normal to each layer can excite a phonon mode [Fig. 1(a)] that creates an equal dipole moment on each layer because of out-of-plane displacement of the $\mathrm{B}$ and $\mathrm{N}$ atoms in opposite directions. The phonon is the $A_{2 \mathrm{u}}$ mode with a wave number around $780 \mathrm{~cm}^{-1}$ ( 23.7 THz) [22,23], with corresponding photon energy around $0.09 \mathrm{eV}$. Figure 1(b) shows a series of 
(a)

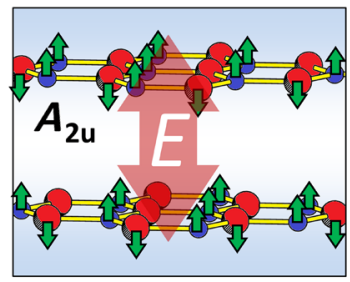

(b)

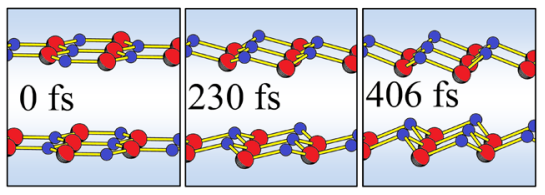

FIG. 1 (color online). Part of the periodic structure of bilayer $h$-BN. Large (red) and small (blue) hatched circles denote boron and nitrogen atoms, respectively. (a) Equilibrium conditions for bilayer $h$-BN. Atomic displacement in the $A_{2 u}$ phonon mode is denoted by small hatched (green) arrows, under optical polarization indicated by (red) arrow labeled $E$. (b) Time evolution under intense infrared laser irradiation with an optical $E$ field of $1 \mathrm{~V} / \AA$, which corresponds to a laser power density of $1.327 \times 10^{13} \mathrm{~W} / \mathrm{cm}^{2}$.

snapshots of the lattice dynamics of the $h$-BN bilayer under the laser $E$ field with an intensity of $1 \mathrm{~V} / \AA$, which corresponds to a power of $1.327 \times 10^{13} \mathrm{~W} / \mathrm{cm}^{2}$. Generation of the parallel dipole moments on each $h$-BN layer causes interlayer attraction.

We can also examine the presence of electronic excitation in addition to the vibrational excitation of the lattice by calculating the electron-ion dynamics. All calculations presented here were performed using time-dependent density functional theory (TDDFT) [24] and TDDFT-molecular dynamics (MD) on a real-time axis within the Ehrenfest approximation [25] as implemented in the FPSEID code $[26,27]$. The split-operator scheme [28,29] was used for time evolution under an alternating $E$ field mimicking laser irradiation [30]. To monitor the real-time electron dynamics, we solved the time-dependent Kohn-Sham (KS) equation

$$
i \hbar \frac{\partial \psi_{\mathrm{n}}^{\mathrm{KS}}(\mathbf{r}, t)}{\partial t}=\left[H^{\mathrm{KS}}(\mathbf{r}, t)+V_{\mathrm{ext}}(\mathbf{r}, t)\right] \psi_{\mathrm{n}}^{\mathrm{KS}}(\mathbf{r}, t),
$$

here, $\psi_{\mathrm{n}}^{\mathrm{KS}}(\mathbf{r}, t)$ is the time-dependent Kohn-Sham orbital, from which the time-dependent charge density, $\rho(\mathbf{r}, t)$, is computed. $H^{\mathrm{KS}}(\mathbf{r}, t)$ expresses the time-dependent KohnSham Hamiltonian, which is uniquely determined as a functional of $\rho(\mathbf{r}, t)$ [24]. The optical $E$ field is described in the length gauge as a gradient of the scalar potential, $V_{\text {ext }}(\mathbf{r}, t)$.

The application and validation of the Ehrenfest approximation have been discussed in previous works [31-33]. For the exchange correlation functional, the local density approximation (LDA) [34] was used for most of the calculations presented here. Some of the results were compared with those within the generalized gradient approximation (GGA) using the Perdew, Burke, and Ernzerhof (PBE) functional [35] with VDW correction
[36] $(\mathrm{PBE}+\mathrm{VDW})$. To express the interaction of the valence electrons with ions, the norm-conserving pseudopotentials [37] were used. Further details of the computational conditions are explained in the Supplemental Material [38].

We provide the results of the simulation with two optical $E$-field intensities of 0.3 and $1 \mathrm{~V} / \AA$. These intensities correspond to laser power densities of $1.194 \times 10^{12}$ and $1.327 \times 10^{13} \mathrm{~W} / \mathrm{cm}^{2}$, respectively. These values should be compared with the currently achievable power of IR lasers that can be tuned to the phonon frequency in this Letter. If the beam spot can be focused to within a $50 \mu$ diameter, the power of the all-solid-state laser devices [41] and the freeelectron laser system $[42,43]$ should be sufficient.

To examine the lattice vibration of the $A_{2 u}$ mode, we first performed a classical MD simulation without an optical $E$ field and with artificial initial velocities on all atoms, such that the lattice oscillation of the out-of-plane $A_{2 u}$ mode was initiated. The calculated wave number of this vibration was $789.44 \mathrm{~cm}^{-1}$ by LDA, and was $768.32 \mathrm{~cm}^{-1}$ by GGA with the PBE functional. These values show fairly good agreement with the reported value of the same mode of the bulk phonon [22,23] from 767 to $783 \mathrm{~cm}^{-1}$. The interlayer interaction was modified with this simulation and PBE + VDW gave different interaction than LDA, and we chose LDA in the present simulation. (See details in section S.II of the Supplemental Material [38].)

Next, we performed the TDDFT-MD simulation with the LDA functional and an optical $E$ field $(0.3 \mathrm{~V} / \AA)$ with a frequency that matches the wave number of $789.44 \mathrm{~cm}^{-1}$. The interlayer distance of the bilayer $h$-BN sheet was initially set as $3.274 \AA$. We started with a planar $h$-BN structure without initial velocities on the ions. The lattice oscillation of the $A_{2 \mathrm{u}}$ mode was induced as shown in Fig. 2(a), which is a plot of the velocities of the B and $N$ atoms in the upper layer in Fig. 1. The velocities in the lower layer are identical and are not shown. Within the displayed time-regime of $1 \mathrm{ps}$, a persistent increase in the amplitude of the oscillating velocities was seen, which means that the frequency with a wave number of $789.44 \mathrm{~cm}^{-1}$ of the optical $E$ field matches the resonance of the $A_{2 \mathrm{u}}$ mode precisely.

We then monitored the interlayer distance, which was defined by the distance between the centers of mass of the $h$-BN layers throughout the TDDFT-MD simulation. By comparing the time evolution of the interlayer distance with and without the optical $E$ field [Fig. 2(b)], a considerable contraction of more than $0.17 \AA$ in 1 ps is seen. (See Sec. III. 1 of the Supplemental Material [38].) After the simulation, we analyzed the electron polarization on each layer from stored electron charge densities at several moments from 21.78 to 972.84 fs. The signs of each polarization, which was computed by the numerical scheme shown in the subsection S. IV. 1 of the Supplemental Material [38], remained the same at all moments indicating a parallel dipole moment on each $h$-BN layer, which seems 
(a) $\left[1 \times 10^{-3}\right]$

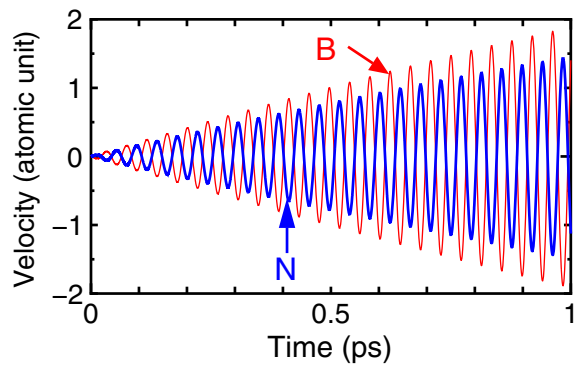

(b)

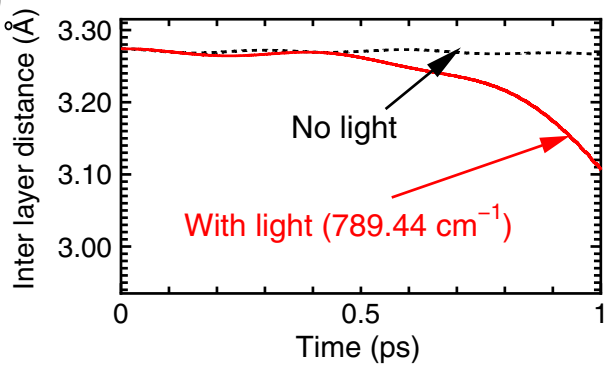

FIG. 2 (color online). (a) Time evolution of the velocities of B and $\mathrm{N}$ atoms on the upper layer of $h-\mathrm{BN}$ as displayed in Fig. 1 in an alternating $E$ field with a maximum intensity of $0.3 \mathrm{~V} / \AA$. The frequency of the $E$ field corresponds to the optical wave number $789.44 \mathrm{~cm}^{-1}$. Thin (red) and thick (blue) lines represent the velocities of the $\mathrm{B}$ and $\mathrm{N}$ atoms, respectively. The positive (negative) velocity denotes the upward (downward) direction normal to the $h$-BN layer in Fig. 1. (b) Distance between centers of mass for each $h$-BN layer as a function of time. The dotted (black) line shows the result without the optical $E$ field, whereas the solid (red) line shows the results with the optical $E$ field, as shown in (a).

to be increased in further long time simulations that require computationally expensive tasks. (See, Sec. III. 2 of the Supplemental Material [38].) To make this effect visible over time scales shorter than $1 \mathrm{ps}$, we performed the same simulations in a higher intensity laser field.

We increased the value of the optical $E$ field to $1 \mathrm{~V} / \AA$ with the same optical frequency of the $A_{2 \mathrm{u}}$ lattice vibration (789.44 $\mathrm{cm}^{-1}$ ), and performed the TDDFT-MD simulation, as displayed as snapshots in Fig. 1(b). The dependence on the frequency of the optical $E$ field was also examined. The interlayer distance is displayed in Fig. 3(a). For the optical frequency resonant with the $A_{2 u}$ lattice vibration, the contraction was large; up to $0.37 \AA$ ( $11.3 \%$ of the original interlayer distance). After $t=430 \mathrm{fs}$, the interlayer distance showed rapid growth toward dissociation of the $h$-BN sheets [Fig. 3(a), $\gamma$ ].

For slightly off-resonance $( \pm 10 \%)$ excitation, the interlayer distance increases toward dissociation of the bilayers after oscillation at higher frequencies [Fig. 3(a), $\alpha$ ], but shows faster contraction at lower frequencies [Fig. 3(a), $\beta$ ]. The frequency dependence of the dynamics of the interlayer distance can be quantified by the electron dynamics, which are represented by the electron polarization in the $z$ direction, defined as
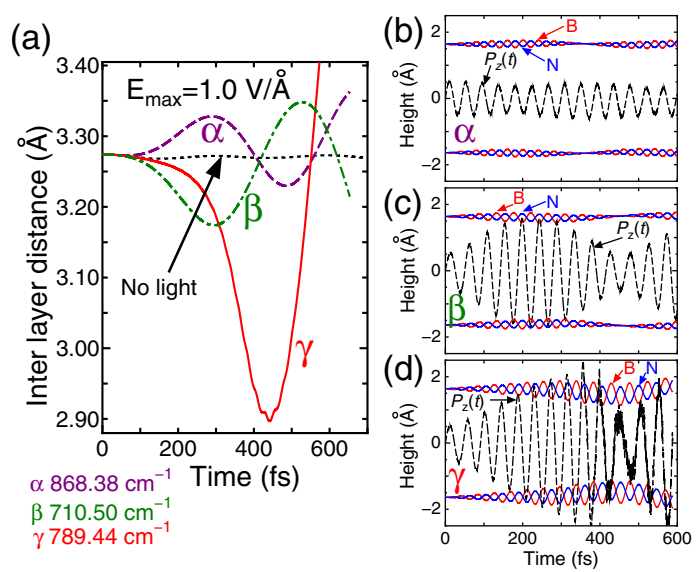

FIG. 3 (color online). Lattice dynamics in bilayer $h$-BN under an optical field with a maximum intensity of $1 \mathrm{~V} / \AA$. (a) Time variation of the interlayer distance under an optical $E$ field with a frequency $10 \%$ higher than the resonant frequency $\left(868.38 \mathrm{~cm}^{-1}\right)$ of the $A_{2 u}$ mode (dashed violet line labeled $\alpha$ ), with a frequency $10 \%$ lower $\left(710.50 \mathrm{~cm}^{-1}\right.$; dotted-dashed green line labeled $\beta$ ), and at the resonant frequency $\left(789.44 \mathrm{~cm}^{-1}\right.$; solid red line labeled $\gamma$ ). The results without irradiation are shown for comparison (dotted black line). Panels (b)-(d) show the time evolution of the heights of the $\mathrm{B}$ and $\mathrm{N}$ atoms of bilayer $h-\mathrm{BN}$ measured from the central position of the two $h$-BN sheets under an optical $E$ field. (b)-(d) correspond to conditions $\alpha, \beta$, and $\gamma$ in (a), respectively. In these panels, the electron polarization from equation (2) are also shown by arrows labeled $P_{\mathrm{z}}(t)$ with an arbitrary common scale.

$$
P_{\mathrm{z}}(t)=\int\left(z-z_{0}\right) \rho(\mathbf{r}, t) d \mathbf{r} .
$$

Here, the integral was performed for the entire unit cell, and $z_{0}$ was set at midpoint between the two $h$-BN sheets. The $z$ component (normal to the $h$-BN sheets) of the time difference of the electron polarization is shown in Figs. 3(b)-3(d) combined with the calculated heights of the $\mathrm{B}$ and $\mathrm{N}$ atoms of the bilayer $h$ - $\mathrm{BN}$, corresponding to cases $\alpha$ to $\gamma$, respectively. For $\alpha$ [868.38 $\mathrm{cm}^{-1}$, Fig. 3(b)], the change in the electron polarization is not large, indicating a minor contribution from the dipole-dipole attraction. However, for $\beta\left[710.50 \mathrm{~cm}^{-1}\right.$, Fig. 3(c)] and $\gamma$ [789.44 $\mathrm{cm}^{-1}$, Fig. 3(d)], the electron polarization is larger, which triggers an initial interlayer contraction. To achieve the interlayer contraction over $10 \%$, the conditions for tuning the optical frequency seem to be narrow. However, current experimental technology does not require this amount of contraction; ultrafast electron-beam diffraction $[15,16]$ can observe contractions of $0.02 \AA$ (less than $1 \%$ ).

The lattice dynamics under an optical resonant $E$ field of $0.3 \mathrm{~V} / \AA$ show synchronization between the lattice vibration and the applied laser for long simulation times of up to 1 ps [Fig. 2(a)]. However, the lattice dynamics with a stronger optical $E$ field of $1 \mathrm{~V} / \AA$ show the phase deviation of the lattice oscillation above $300 \mathrm{fs}$ [Fig. 3(d)], later than this time, the contraction turned to expansion. The electron polarization shows noisy behavior faster than lattice motion 
during expansion [Fig. 3(d)], which is associated with electronic excitation as discussed below.

Figure 4 shows a plot of the total energy (solid line) throughout the TDDFT-MD simulation calculated with the same density functional theory (DFT) formalisms [44] as for $\gamma$ with a conserved quantity obtained by the formalisms in Ref. [45] (dashed horizontal line) that justifies the numerical stability of the current simulation. Meanwhile, the series of the static DFT total energies with atomic coordinates at the moments when the optical $E$ field is zero, the vertical lines in Fig. 4(a), are computed as shown by (blue) dots. Up to $350 \mathrm{fs}$, the (blue) dots are on solid (red) line, indicating that the TDDFT-MD simulation follows the Born-Oppenheimer surface of the electronic ground state. Thus, the increase in the series of the static DFT total energy up to $350 \mathrm{fs}$ is an increase in the amplitude of the lattice vibration, namely, the classical representation of the phonon excitation. Further analysis is explained in the Supplemental Material [38], see subsection S. III. 3.

After $350 \mathrm{fs}$, the static DFT total energies are smaller than the total energies obtained by the TDDFT-MD simulation with the same atomic coordinates. The deviation of the TDDFT total energies compared with those calculated by static DFT means the electronic excitation,

During electronic excitation, the opposite dipole inside the $h$-BN layers was induced that reduced the interlayer attraction, see subsection S. IV. 2 in the Supplemental Material [38]. Because the photon energy of $0.09 \mathrm{eV}$ is much smaller than the intrinsic band gap of $h-\mathrm{BN}(\sim 4.0 \mathrm{eV}$

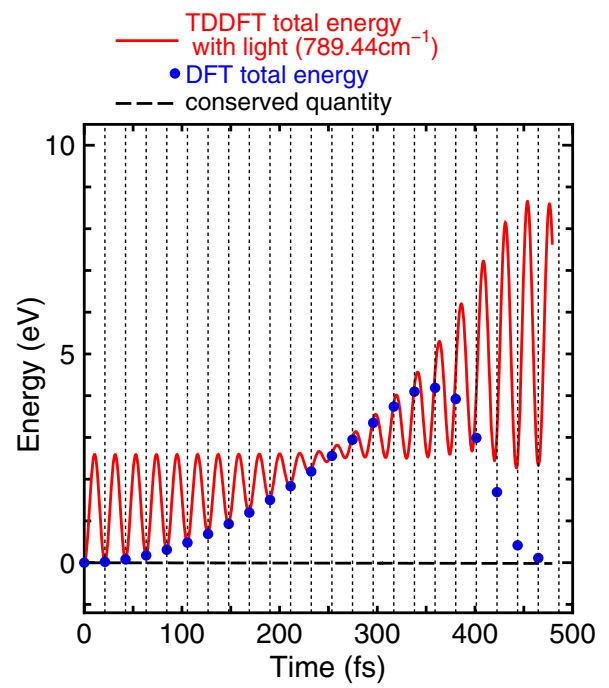

FIG. 4 (color online). The solid (red) line shows a calculated total energy for the TDDFT-MD simulation with the conditions shown in Fig. 3(a) $\gamma$ and Fig. 3(d). The origin of the energy is set to the value at the beginning of the simulation. The horizontal dashed (black) line shows the conserved quantity, which was obtained using the scheme shown in Ref. [45]. The vertical dotted lines show the moments when the optical $E$ field is zero, corresponding to half of the period of the $A_{2 \mathrm{u}}$ mode oscillation. The blue dots are the total energy of the static DFT calculation at moments denoted by the vertical dotted lines. by LDA calculations), the origin of the electronic excitation can either be due to multiphoton excitation [46] or to narrowing of the band gap, see section S. V. of the Supplemental Material [38], during the increase in the amplitude of the lattice vibration. Here, we note that the decreasing of the gap can reduce excitonic binding energy due to an increase of the screening. Beside optical excitation, an excitation by the large amplitude of phonon should also be taken into account for increase of the total energy by the amount of band gap. The electronic excitation by ionic motion was reported by TDDFT calculations [47-49].

The electron beam diffraction under femtosecond laser illumination $[15,16]$ detected a subpicometer $(0.01 \AA)$ contraction of graphite in the time range of $0.2-2.5$ ps. These experiments suggest the feasibility of observing a contraction of $0.17 \AA$ within 1 ps [Fig. 2(b)]. Then the retention time of the contracted geometry after turning off the IR laser should be an experimental question. To answer this, performing the simulation after turning off the IR laser irradiation requires a new technique to avoid artificial high-frequency excitation [50]. However, we estimated the retention time as $190 \mathrm{fs}$ from the simulation giving ions' velocities comparable to those shown in Fig. 2(a) without an optical $E$ field, see, again, section S. II of the Supplemental Material [38].

From current simulations, we know that too strong an $E$ field shows a rapid change from contraction to expansion, which was also the case for femtosecond laser illumination $[15,16]$. To avoid expansion, tuning the intensity of the $E$ field to less than $1 \mathrm{~V} / \AA$ (with power less than $1.327 \times 10^{13} \mathrm{~W} / \mathrm{cm}^{2}$ ) would be required. Otherwise, we should lower the frequency of the IR laser like the chirp technique [51], since the lattice dynamics in Fig. 3(a) show the decreases its vibrational frequency after $350 \mathrm{fs}$. The lowering IR frequency would maintain the contraction over a longer time.

Finally, we note that the interlayer distance could be modified after intercalating. So far, for $h$-BN, possible experiment with use of the same equipment as Ref. [52] seems to be promising. This is common in other layered material such as transition metal dichalcogenides, yet the functional of DFT is a matter for proper interlayer interactions as well as long-time simulation suitable for a heavier mass of elements. Instead of using mechanical devices for compression, the laser-induced interlayer contraction could produce high-pressure conditions between the atomic layers and induce new phases of intercalated molecules.

All calculations were performed by using the Parallel Computing System in AIST. Y. M. and T. M. thank M. Kakehata, D. Yoshitomi, and K. Torizuka for fruitful discussions on accessibility of the studied conditions by experimental equipment. Y. M. acknowledges the support by MEXT Grants-in-Aid for Scientific Research on Innovative Areas "Science of Atomic Layers" (Projects No. 2506, No. 26107534). H. Z. acknowledges financial support from the National Natural Science Foundation of China (NSFC. Grant No. 11474207). A. R. acknowledges 
the financial support from the European Research Council Advanced Grant DYNamo (No. ERC-2010- AdG-267374), Spanish Grant (No. FIS2013-46159-C3-1-P), Grupos Consolidados UPV/EHU del Gobierno Vasco (No. IT578-13), and European Community FP7 Project CRONOS (Grant No. 280879-2), and COST Actions Grants No. CM1204 (XLIC) and No. MP1306 (EUSpec).

*hongzhang@scu.edu.cn

[1] K. S. Novoselov, A. K. Geim, S. V. Morozov, D. Jiang, M. I. Katsnelson, I. V. Grigorieva, S. V. Dubonos, and A. A. Firsov, Nature (London) 438, 197 (2005).

[2] W.-Q. Han, L. Wu, Y. Zhu, K. Watanabe, and T. Taniguchi, Appl. Phys. Lett. 93, 223103 (2008).

[3] A. Splendiani, L. Sun, Y. Zhang, T. Li, J. Kim, C.-Y. Chim, G. Galli, and F. Wang, Nano Lett. 10, 1271 (2010).

[4] S. Jeong, D. Yoo, J. Jang, M. Kim, and J. Cheon, J. Am. Chem. Soc. 134, 18233 (2012).

[5] Y.-H. Lee, X.-Q. Zhang, W. Zhang, M.-T. Chang, C.-T. Lin, K.-D. Chang, Y.-C. Yu, J. T.-W. Wang, C.-S. Chang, L.-J. Li et al., Adv. Mater. 24, 2320 (2012).

[6] Q. Tang and Z. Zhou, Prog. Mater. Sci. 58, 1244 (2013).

[7] Y.-M. Lin, K. A. Jenkins, A. Valdes-Garcia, J. P. Small, D. B. Farmer, and P. Avouris, Nano Lett. 9, 422 (2009).

[8] M. Fontana1, T. Deppe, A. K. Boyd, M. Rinzan, A. Y. Liu, M. Paranjape, and P. Barbara, Sci. Rep. 3, 1634 (2013).

[9] L. Britnell, R. V. Gorbachev, R. Jalil, B. D. Belle, F. Schedin, M. I. Katsnelson, L. Eaves, S. V. Morozov, A. S. Mayorov, N. M. R. Peres et al., Nano Lett. 12, 1707 (2012).

[10] G. Giovannetti, P. A. Khomyakov, G. Brocks, P. J. Kelly, and J. van den Brink, Phys. Rev. B 76, 073103 (2007).

[11] C. R. Dean, A. F. Young, I. Meric, C. Lee, L. Wang, S. Sorgenfre, K. Watanabe, T. Taniguchi, P. Kim, K. L. Shepard et al., Nat. Nanotechnol. 5, 722 (2010).

[12] L. Britnell, R. V. Gorbachev, R. Jalil, B. D. Belle, F. Schedin, A. Mishchenko, T. Georgiou, M. I. Katsnelson, L. Eaves, S. V. Morozov et al., Science 335, 947 (2012).

[13] M. P. Levendorf, C.-J. Kim, L. Brown, P. Y. Huang, R. W. Havener, D. A. Muller, and J. Park, Nature (London) 488, 627 (2012).

[14] A. K. Geim and I. V. Grigorieva, Nature (London) 499, 419 (2012).

[15] F.Carbone, P. Baum, P. Rudolf, and A. H. Zewail, Phys. Rev. Lett. 100, 035501 (2008).

[16] R. K. Raman, Y. Murooka, C.-Y. Ruan, T. Yang, S. Berber, and D. Tománek, Phys. Rev. Lett. 101, 077401 (2008).

[17] Y. Miyamoto, H. Zhang, and D. Tománek, Phys. Rev. Lett. 104, 208302 (2010).

[18] T. Kanayama, Jpn. J. Appl. Phys. 33, L1792 (1994).

[19] Y. Shimotsuma, K. Hirao, P. G. Kazansky, and J. Qiu, Jpn. J. Appl. Phys. 44, 4735 (2005).

[20] A. Rousse, C. Rischel, S. Fourmaux, I. Uschmann, S. Sebban, G. Grillon, P. Balcou, E. Förster, J. P. Geindre, P. Audebert et al., Nature (London) 410, 65 (2001).

[21] Y. Miyamoto, T. Miyazaki, A. Rubio, and H. Zhang, Appl. Phys. Lett. 104, 201107 (2014).

[22] R. Geick, C. H. Perry, and G. Rupprecht, Phys. Rev. 146, 543 (1966).

[23] K. H. Michel and B. Verberck, Phys. Rev. B 83, 115328 (2011).
[24] E.Runge and E. K. U. Gross, Phys. Rev. Lett. 52, 997 (1984).

[25] P. Ehrenfest, Z. Phys. 45, 455 (1927).

[26] O. Sugino and Y. Miyamoto, Phys. Rev. B 59, 2579 (1999).

[27] O. Sugino and Y. Miyamoto, Phys. Rev. B 66, 089901(E) (2002).

[28] M. Suzuki, J. Phys. Soc. Jpn. 61, 3015 (1992).

[29] M. Suzuki and T. Yamauchi, J. Math. Phys. (N.Y.) 34, 4892 (1993).

[30] A. Castro, M. A. L. Marques, J. A. Alonso, G. F. Bertsch, and A. Rubio, Eur. Phys. J. D 28, 211 (2004).

[31] J. L. Alonso, X. Andrade, P. Echenique, F. Falceto, D. PradaGracia, and A. Rubio, Phys. Rev. Lett. 101, 096403 (2008).

[32] X. Andrade, A. Castro, D. Zueco, J. L. Alonso, P. Echenique, F. Falceto, and A. Rubio, J. Chem. Theory Comput. 5, 728 (2009).

[33] Fundamentals of time-dependent density functional theory, edited by M. A. L. Marques, N. T. Maitra, F. M. S. Nogueira, E. K. U. Gross, and A. Rubio, Lecture Note of Physics, Vol. 837 (Springer, Berlin, 2012).

[34] J. P. Perdew and A. Zunger, Phys. Rev. B 23, 5048 (1981).

[35] J. P. Perdew, K. Burke, and M. Ernzerhof, Phys. Rev. Lett. 77, 3865 (1996).

[36] A. Tkatchenko and M. Scheffler, Phys. Rev. Lett. 102, 073005 (2009).

[37] N. Troullier and J. L. Martins, Phys. Rev. B 43, 1993 (1991).

[38] See Supplemental Material at http://link.aps.org/ supplemental/10.1103/PhysRevLett.114.116102 for numerical conditions and precisions, which includes Refs. $[34-36,39,40]$.

[39] N. Marom, A. Tkatchenko, M. Rossi, V. V. Gobre, O. Hod, M. Scheffler, and L. Kronik, J. Chem. Theory Comput. 7, 3944 (2011).

[40] A. Marini, P. García-González, and A. Rubio, Phys. Rev. Lett. 96, 136404 (2006).

[41] I. M. Bayanov, R. Danielius, P. Heinz, and A. Seilmeier, Opt. Commun. 113, 99 (1994).

[42] R. A. Kaindl, D. C. Smith, M. Joschko, M. P. Hasselbeck, M. Woerner, and T. Elsaesser, Opt. Lett. 23, 861 (1998).

[43] T. Imai, K. Tsukiyama, T. Shidara, M. Yoshida, T. Morotomi, and K. Hisazumi, in Proceedings of the 2010 International Particle Accelerator Conference, IPAC'10 (Kyoto, Japan, 2010) (IEEE, Piscataway, NJ, 2010), Vol. 10.

[44] J. Ihm, A. Zunger, and M. L. Cohen, J. Phys. 12, 4409 (1979).

[45] Y. Miyamoto and H. Zhang, Phys. Rev. B 77, 165123 (2008).

[46] Here, we must note that the excitation with the aid of integer numbers of photons cannot correctly be expressed by current semiclassical simulation representing the laser as an alternating $E$ field.

[47] M. Lindenblatt and E. Pehlke, Phys. Rev. Lett. 97, 216101 (2006)

[48] M. Lindenblatt, J. van Heys, and E. Pehlke, Surf. Sci. 600, 3624 (2006).

[49] A. V. Krasheninnikov, Y. Miyamoto, and D. Tománek, Phys. Rev. Lett. 99, 016104 (2007).

[50] Abrupt turning off causes induction of higher frequency on the optical $E$ field, which causes artificial excitation on the system. To avoid this, gradual attenuation of optical amplitude is necessary, and how slow the attenuation should be is for future work.

[51] D. Strickland and G. Mourou, Opt. Commun. 55, 447 (1985).

[52] S. Dai, Z. Fei, Q. Ma, A. S. Rodin, M. Wagner, A. S. McLeod, M. K. Liu, W. Gannett, W. Regan, K. Watanabe et al., Science 343, 1125 (2014). 